\title{
Características produtivas e de qualidade de carne de frangos de corte submetidos a dietas de terminação sem suplemento vitamínico- mineral ou calcário e fosfato bicálcico e submetidos a estresse térmico pré-abate
}

\section{Characteristics productive and meat quality of broiler chickens fed finisher diet without vitamin-mineral supplement or limestone and dicalcium phosphate and submitted to heat stress pre-slaughter}

\author{
Alexandre Oba ${ }^{1 *}$; João Waine Pinheiro ${ }^{1}$; Caio Abércio da Silva ${ }^{1}$; \\ Mara Regina Stipp Balarin²; Francielle Renata Bueno³; Maurício de Almeida ${ }^{3}$; \\ Rimena Vircelino do Amaral ${ }^{4}$; Sandra Regina Brunelli ${ }^{5}$
}

\section{Resumo}

\begin{abstract}
Buscar mecanismos que possam reduzir o custo de produção das aves, sem reduzir o desempenho e a qualidade do produto final é um importante fator dentro da cadeia avícola. Desta forma, o objetivo deste trabalho foi avaliar a retirada do suplemento vitamínico-mineral ou calcário e fosfato bicálcico da ração na fase de acabamento, além de submeter os frangos de corte a estresse térmico pré-abate visando avaliar o desempenho e a qualidade de carne do peito. Foram utilizados 108 frangos de corte machos da linhagem Hybro com 42 dias de idade, que receberam uma ração controle, ração sem suplemento vitamínico-mineral e sem calcário e fosfato bicálcico, dos 42 aos 49 dias de idade. Foram avaliados os dados de desempenho, rendimento de carcaça e cortes, bioquímica do sangue e qualidade de carne. Os resultados mostraram que a retirada do suplemento vitamínico-mineral ou o calcário e o fosfato bicálcico da dieta de terminação não prejudicou o desempenho, características de carcaça e qualidade de carne, mostrando assim como um possível mecanismo para reduzir os custos de produção. Já o estresse térmico quente no pré-abate proporcionou uma piora na qualidade de carne das aves.
\end{abstract}

Palavras-chave: Aves, cor, desempenho, dieta terminação, $\mathrm{pH}$

\begin{abstract}
Search mechanisms that can reduce the cost of production of poultry is an important factor in the poultry industry. Thus the objective of this study was to evaluate the removal of vitamin and mineral supplement or limestone and dicalcium phosphate feed during the finishing phase, further of the broilers chickens to heat stress pre-slaughter. Hybro male broiler $(n=108)$ aged 42 days old were evaluated until 49 days old to evaluate the performance, carcass and cuts yield, blood biochemistry and meat quality.
\end{abstract}

\footnotetext{
${ }^{1}$ Profs. Drs. do Dept ${ }^{\mathrm{o}}$ de Zootecnia, Universidade Estadual de Londrina UEL, Londrina, PR. E-mail: oba@uel.br; jwaine@uel.br; casilva@uel.br

${ }^{2}$ Prof $^{\mathrm{a}} \mathrm{Dr}^{\mathrm{a}}$. do Dept ${ }^{\circ}$ de Medicina Veterinária Preventiva, UEL, Londrina, PR. E-mail: marastipp@uol.com.br

${ }^{3}$ Discente(s) de Pós-graduação em Ciência Animal, UEL, Londrina, PR. E-mail: francielle_bueno89@hotmail.com; mauricio_ alm_jn@hotmail.com

${ }^{4}$ Discente de Pós-graduação em Engenharia Agrícola da Feagri, Universidade Estadual de Campinas, UNICAMP, Campinas, SP. E-mail: rima_vercellino@yahoo.com.br

${ }^{5}$ Prof $^{\mathrm{a}} \mathrm{Dr}^{\mathrm{a}}$ da Faculdade de Ciências Sociais e Agrárias de Itapeva, FAIAT, Itapeva, SP. E-mail: sandrabrunelli@dilk.com.br

* Autor para correspondência
} 
The results showed that the removal of vitamin and mineral supplement or limestone and dicalcium phosphate feed during the finishing phase did not impair the performance, carcass characteristics and meat quality, as well as showing a possible mechanism to reduce production costs. While heat stress pre-slaughter provided a poor meat quality of poultry.

Key words: Color, finishing phase, performance, $\mathrm{pH}$, poultry

\section{Introdução}

A avicultura mundial e a brasileira têm alcançado índices zootécnicos excelentes, com aves ganhando peso rapidamente e uma excelente conversão alimentar. Mesmo assim a indústria avícola vem almejando por meio de pesquisas a redução dos custos de produção. A ração representa aproximadamente de 70 a $80 \%$ deste custo. Assim, qualquer redução no valor do custo com a nutrição das aves, representa muito em termos globais. Desta forma, nutricionistas vêm testando alimentos alternativos, aditivos, redução de nutrientes e até mesmo a retirada de outros, com a finalidade de reduzir os custos, sem prejudicar o desempenho e qualidade do produto final.

Com o intuito de reduzir o custo de produção, pesquisas têm retirado o suplemento vitamínicomineral das rações, sem que ocorram prejuízos no desempenho das aves, visto que esta retirada normalmente é na dieta de terminação, que corresponde a um período curto, e como as aves têm reservas de minerais e vitaminas lipossolúveis, pode ser que não ocorra prejuízos no desempenho das aves. Resultados positivos quanto a retira do suplemento vitamínico-mineral foram obtidos para todos os índices produtivos (SKINNER; WALDROUP; WALDROUP, 1992; CHRISTMAS; HARMS; SLOAN, 1995). Enquanto que Maiorka et al. (2002) observaram piora na conversão alimentar, Khajali, Asadi e Zamani (2006) detectaram um menor consumo de ração e Moravej et al. (2012) observaram que somente a retirada de $100 \%$ do suplemento vitamínico-mineral proporcionou menor ganho de peso das aves.

É sabido que vitamina E (OLIVO et al., 2001) e selênio (PISSINATI et al., 2012) melhoraram a qualidade da carne das aves, pois atuam como antioxidantes, preservando assim a integridade da membrana celular. Porem estes podem ser armazenados no organismo animal e dependendo do período de restrição, pode não proporcionar efeitos prejudiciais no desempenho e qualidade de carne.

O cálcio e o fósforo são minerais importantes na nutrição animal, por serem necessários não apenas para a ótima taxa de crescimento, mas também para a mineralização óssea, e estarem envolvidos em grande número de processos fisiológicos, como transmissão de impulsos nervosos, contração muscular, coagulação sanguínea e ativação de sistemas enzimáticos (SÁ et al., 2004).

Nas aves, o cálcio é encontrado quase que sua totalidade (99\%) no esqueleto ósseo e segundo Scheideler et al. (1995), os frangos de corte conseguem ganhar peso eficientemente com relativa amplitude de variação dos níveis de cálcio e fósforo dietéticos. Isto ocorre em função da grande reserva destes minerais nos ossos.

Além de atuar nas reações metabólicas para o desenvolvimento dos animais, o cálcio e o fósforo estão intimamente relacionados com o processo de rigor mortis que ocorre na transformação do músculo em carne, em que estes são responsáveis pelo metabolismo energético e pela contração muscular, respectivamente. Fatores estes que influenciam na qualidade da carne.

Mediante ao exposto, este trabalho teve por objetivo avaliar o desempenho, rendimento de carcaça e cortes, qualidade de carne e bioquímica do sangue de frangos alimentados com dietas sem a inclusão de suplemento vitamínico-mineral ou sem calcário e fosfato bicálcico na fase de acabamento, além da ação do estresse térmico pré-abate. 


\section{Material e Métodos}

Foram utilizados 108 frangos de corte machos da linhagem Hybro com 42 dias de idade. Os frangos foram selecionados com peso médio de 2940 g. Estes receberam manejos conforme práticas comerciais, com água e alimento ad libitum durante o período experimental de 7 dias, que correspondeu dos 42 aos 49 dias de idade das aves.

As dietas experimentais foram formuladas à base de milho e farelo de soja, sendo isoproteicas e isoenergéticas, e todos os nutrientes atendiam às exigências de Rostagno et al. (2005), exceto para os níveis de vitaminas e microminerais, cálcio e fósforo que não atendiam estas exigências, visto que estes variavam em função do tratamento experimental. Os níveis de cálcio foram $(0,73$; $0,73$ e $0,10 \%)$ e fósforo disponível $(0,36 ; 0,36$ e $0,10 \%$, respectivamente para as dietas controle, sem suplemento vitaminico-mineral, e sem fosfato bicálcico e calcário (Tabela 1).

Tabela 1. Composição nutricional e valor energético das rações experimentais.

\begin{tabular}{lccc}
\hline \multirow{2}{*}{ Ingredientes } & \multicolumn{3}{c}{ Ração } \\
\cline { 2 - 4 } & Controle & $\begin{array}{c}\text { Sem suplemento } \\
\text { vitamínico mineral }\end{array}$ & $\begin{array}{c}\text { Sem fosfato bicálcico e } \\
\text { calcário }\end{array}$ \\
\hline Milho & 68,36 & 69,20 & 73,14 \\
Farelo de soja & 24,12 & 23,96 & 23,19 \\
Óleo de soja & 3,97 & 3,69 & 2,38 \\
Fosfato bicálcico & 1,80 & 1,80 & 0,00 \\
Calcário & 0,47 & 0,47 & 0,00 \\
Supl. Vitamínico mineral ${ }^{1}$ & 0,40 & 0,00 & 0,40 \\
Sal & 0,38 & 0,38 & 0,38 \\
L-lisina & 0,29 & 0,29 & 0,30 \\
DL-metionina & 0,21 & 0,21 & 0,21 \\
Composição Calculada & & & \\
Energ. Metab (kcal/kg) & $3.200,00$ & $3.200,00$ & $3.200,00$ \\
Proteína bruta (\%) & 17,24 & 17,24 & 17,24 \\
Cálcio (\%) & 0,73 & 0,73 & 0,10 \\
Fósforo disponível (\%) & 0,36 & 0,36 & 0,10 \\
Lisina dig. (\%) & 0,97 & 0,97 & 0,97 \\
Metionina (\%) & 0,46 & 0,46 & 0,45 \\
Met+cistina (\%) & 0,70 & 0,70 & 0,70 \\
\hline
\end{tabular}

${ }^{1}$ Composição suplemento vitamínico mineral: iodo $152,34(\mathrm{mg} / \mathrm{kg})$, selênio 46,87 (mg/kg), manganês $10.898(\mathrm{mg} / \mathrm{kg})$, zinco 9.570 $(\mathrm{mg} / \mathrm{kg})$, ferro $6.562(\mathrm{mg} / \mathrm{kg})$, cobre $1.406(\mathrm{mg} / \mathrm{kg})$, cobalto $31,25(\mathrm{mg} / \mathrm{kg})$, vit A $2.250 .000(\mathrm{UI} / \mathrm{kg})$, vit D $630(\mathrm{UI} / \mathrm{kg})$, vit E 2.000 $(\mathrm{mg} / \mathrm{kg})$, vit K $504(\mathrm{mg} / \mathrm{kg})$, vit B1 $1.393(\mathrm{mg} / \mathrm{kg})$, vit B2 $1.000(\mathrm{mg} / \mathrm{kg})$, vit B6 $263(\mathrm{mg} / \mathrm{kg})$, vit B12 $2.500(\mathrm{mg} / \mathrm{kg})$, pantotenato de cálcio $2.019(\mathrm{mg} / \mathrm{kg})$, niacina $5.007(\mathrm{mg} / \mathrm{kg})$, biotina $3,12(\mathrm{mg} / \mathrm{kg})$, ácido fólico $63(\mathrm{mg} / \mathrm{kg})$, colina $70.000(\mathrm{mg} / \mathrm{kg})$, antioxidante $2.500(\mathrm{mg} / \mathrm{kg})$, veiculo Q.S.P. $1000 \mathrm{~g}$.

Fonte: Elaboração dos autores.

Os tratamentos experimentais consistiam em uma dieta controle, uma dieta sem a inclusão de suplemento vitamínico-mineral, e uma dieta sem a inclusão de calcário e fosfato bicálcico. Para os dados de desempenho foi utilizado um delineamento inteiramente casualizado, com três tratamentos e seis repetições, com 6 aves por unidade experimental. Já para o rendimento de carcaça e cortes foi adotado um delineamento inteiramente ao acaso, com três tratamentos, seis repetições com duas aves por parcela. Para as analises de bioquímica do sangue e qualidade de carne foi adotado um delineamento inteiramente casualizado, em esquema fatorial $3 \mathrm{x}$ 2 (dietas de terminação $\mathrm{x}$ estresse pré-abate), com 
seis tratamentos e seis repetições de duas aves por parcela experimental.

No final do período experimental, após um período de jejum de 10 horas, foi aplicado estresse térmico quente, que consistiu em deixar as aves por 30 minutos em câmara térmica a $35^{\circ} \mathrm{C}$. Em seguida, foram coletadas amostras de sangue através de punção da veia braquial da asa. Após obtenção do soro, estes foram congelados a $-20^{\circ} \mathrm{C}$ até o momento das análises de cálcio, fósforo, ferro, colesterol e triglicérides. Após esta coleta, duas aves por parcela experimental foram abatidas, conforme práticas comerciais, com insensibilização elétrica através do aparelho da marca Fluxo, modelo FX 2.0, no qual as aves foram expostas por 10 segundos a $42 \mathrm{v}$ e $800 \mathrm{~Hz}$. Em seguida foram sangradas, escaldadas, evisceradas e resfriadas.

O rendimento de carcaça foi considerado a carcaça eviscerada, sem cabeça + pescoço e pés, em relação ao peso de abate. Já o rendimento de cortes (peito, coxa+sobrecoxa, dorso e asas) foi considerado em relação ao peso da carcaça eviscerada.

Após o abate foi coletado o músculo Pectorales major, onde se determinou $\mathrm{pH}$ inicial após 20 minutos do abate, através de potenciômetro de contato da marca Testo, modelo 205. Em seguida as amostras foram armazenadas a $4^{\circ} \mathrm{C}$ por 24 horas para se determinar as demais análises, como $\mathrm{pH}$ final, coloração (medido na parte ventral do músculo, através de colorímetro Minolta CR10, onde foram avaliados os componentes $\mathrm{L}^{*}$ (luminosidade), $\mathrm{a}^{*}$ (componente vermelho-verde) e $b^{*}$ (componente amarelo-azul), que foram expressos no sistema de cor CIELAB), capacidade de retenção de água segundo Barbut (1996), perdas no cozimento (CASON; LYON; PAPA, 1997) e força cisalhamento que foi determinado em aparelho Texture Analyser TA-XT2, utilizando a sonda Warner-Bratzler.

\section{Resultados e Discussão}

Os resultados apresentados na Tabela 2 mostram que não houve efeito da retirada do suplemento vitamínico-mineral ou do fosfato bicálcico e calcário da ração de frangos de corte dos 42 aos 49 dias de idade, sobre o desempenho e rendimento de carcaça e cortes de frangos. Estes resultados concordam com os observados por Sayadi et al. (2005), que relatam que a retirada do suplemento vitamínico-mineral nas duas últimas semanas não interfere nos índices produtivos, porém discorda de Maiorka et al. (2002) que verificaram piora na conversão alimentar ao retirar da dieta o suplemento vitamínico-mineral aos 42 e 45 dias, sendo que as aves foram abatidas aos 49 dias.

Tabela 2. Desempenho e rendimento de carcaça e cortes de frangos de 42 a 49 dias de idade, que foram alimentados com diferentes dietas.

\begin{tabular}{lcccc}
\hline \multirow{2}{*}{ Características } & \multicolumn{3}{c}{ Ração } & CV (\%) \\
\cline { 2 - 4 } & Controle & $\begin{array}{c}\text { Sem suplemento } \\
\text { vitamínico mineral }\end{array}$ & $\begin{array}{c}\text { Sem fosfato bicálcico e } \\
\text { calcário }\end{array}$ & CV \\
\hline Ganho peso (g) & 577 & 544 & 585 & 11,36 \\
Consumo ração (g) & 1282 & 1192 & 1267 & 9,27 \\
Conv. Alimentar & 2,23 & 2,19 & 2,17 & 5,49 \\
Rend. carcaça (\%) & 73,13 & 73,54 & 73,38 & 2,03 \\
Rend. peito (\%) & 34,57 & 34,39 & 34,29 & 3,95 \\
Ren. coxa+sobrecoxa (\%) & 34,11 & 34,20 & 34,37 & 3,28 \\
Rend. asa (\%) & 11,36 & 11,59 & 11,34 & 4,41 \\
Rend. dorso (\%) & 19,96 & 19,82 & 19,99 & 4,64 \\
\hline
\end{tabular}

Fonte: Elaboração dos autores. 
O desempenho semelhante das aves com dietas sem fosfato bicálcico e calcário provavelmente foi em função do grande depósito destes minerais nos ossos. Desse modo o organismo mobilizou estes minerais dos ossos, proporcionando assim uma homeostase sanguínea (Tabela 3), o que levou a manutenção do desempenho das aves.

Tabela 3. Resultados de cálcio, fósforo e ferro no sangue de frangos alimentados com diferentes dietas de 42 a 49 dias de idade e submetidos ou não ao estresse térmico pré-abate.

\begin{tabular}{|c|c|c|c|}
\hline \multirow{2}{*}{ Tratamentos } & \multicolumn{3}{|c|}{ Níveis de minerais no sangue (mg/dL) } \\
\hline & $\mathrm{Ca}$ & $\mathrm{P}$ & $\mathrm{Fe}$ \\
\hline \multicolumn{4}{|l|}{ Tipos ração $(\mathrm{R})$} \\
\hline Controle & 9,23 & 5,93 & $269 b$ \\
\hline Sem supl.vitamínico mineral & 8,68 & 5,73 & $368 \mathrm{a}$ \\
\hline Sem fosfato bicálcio e calcário & 10,17 & 5,40 & $435 \mathrm{a}$ \\
\hline Teste F & $0,98 \mathrm{NS}$ & $0,76 \mathrm{NS}$ & $11,81 * *$ \\
\hline DMS & 2,30 & 0,88 & 85,10 \\
\hline \multicolumn{4}{|l|}{ Manejo pré-abate (E) } \\
\hline Sem estresse & 9,36 & 5,69 & 353 \\
\hline Com estresse & 9,72 & 5,86 & 362 \\
\hline Teste F & $0,22^{\mathrm{NS}}$ & $0,33^{\mathrm{NS}}$ & $0,09^{\mathrm{NS}}$ \\
\hline DMS & 1,55 & 0,60 & 57,54 \\
\hline F interação R x E & $0,33^{\mathrm{NS}}$ & $0,51^{\mathrm{NS}}$ & $1,16^{\mathrm{NS}}$ \\
\hline Coef. Variação (\%) & 23,93 & 15,18 & 23,65 \\
\hline
\end{tabular}

$\mathrm{Na}$ mesma coluna, médias seguidas de letras diferentes, indicam diferenças estatísticas pelo teste de Tukey $(\mathrm{P}<0,05) ; \mathrm{DMS}=$ diferença mínima significativa.

Fonte: Elaboração dos autores.

Quanto à retirada do suplemento vitamínicomineral, estes também não influenciaram no desempenho animal, visto que os microminerais também podem estar armazenados nos ossos e tecidos e como suas exigências são pequenas e o período de tempo de retirada foi curto, as aves provavelmente também mobilizaram estes para garantir suas funções metabólicas e não prejudicar o desempenho das aves. Já com a falta de vitaminas presentes no suplemento, também pode ter ocorrido o mesmo motivo, visto que as vitaminas lipossolúveis podem ser armazenadas nos tecidos e devido ao período curto de restrição destas, não proporcionou perdas no desempenho.

Os resultados de rendimento de carcaça e cortes dos frangos alimentados com dietas sem suplemento vitamínico-mineral ou fosfato bicálcico e calcário, também não apresentaram diferenças significativas. Resultados esperados, visto que estes tratamentos não influenciaram no desempenho das aves.

Pode ser observado também na Tabela 3, que os níveis sanguíneos de ferro nos tratamentos sem suplemento vitamínico-mineral e sem fosfato bicálcico e calcário foram superiores ao tratamento controle. Isto pode ter ocorrido, pois a absorção de ferro é influenciada por outros cátions como o cálcio. Estudos realizados mostraram que o cálcio chega a reduzir a absorção de ferro em até $60 \%$ (HALLBERG, et al., 1992).

$\mathrm{O}$ estresse térmico quente pré-abate não influenciou $(\mathrm{P}>0,05)$ nos níveis sanguíneos de cálcio, fósforo e ferro. Mostrando assim, que as concentrações destes importantes minerais não se alteram devido ao estresse térmico quente pré-abate. Com relação aos níveis de colesterol e triglicérides no sangue de frangos alimentados com os diferentes rações, não foi observado diferença significativa. O mesmo pode ser observado quando se avaliou a aplicação de estresse no manejo pré-abate (Tabela 4). Não foi observado interação entre os tipos de rações e o manejos pré-abates para os valores de bioquímica do sangue, 
Tabela 4. Resultados de colesterol, triglicérides e uréia no sangue de frangos alimentados com diferentes dietas e submetidos ou não ao estresse térmico pré-abate.

\begin{tabular}{lcc}
\hline Tratamentos & Colesterol (mg/dL) & Triglicerídes (mg/dL) \\
\hline Tipo de ração (R) & & \\
Controle & 131 & 50,67 \\
Sem suplemento vitamínico mineral & 122 & 61,67 \\
Sem fosfato bicálcio e calcário & 140 & 60,67 \\
\hline Teste F & $1,07^{\mathrm{NS}}$ & $1,27^{\mathrm{NS}}$ \\
DMS & 30,58 & 18,84 \\
\hline Manejo pré-abate (E) & 128 & 57,05 \\
Sem estresse & 134 & 58,28 \\
Com estresse & $0,31^{\mathrm{NS}}$ & $0,04^{\mathrm{NS}}$ \\
\hline Teste F & 20,68 & 12,74 \\
DMS & $2,26^{\mathrm{NS}}$ & $0,16^{\mathrm{NS}}$ \\
\hline F interação R x E & 23,22 & 32,43 \\
\hline Coef. Variação (\%) &
\end{tabular}

$\mathrm{Na}$ mesma coluna, médias seguidas de letras diferentes, indicam diferenças estatísticas pelo teste de Tukey $(\mathrm{P}<0,05)$; DMS = diferença mínima significativa.

Fonte: Elaboração dos autores.

Em relação à qualidade de carne, os resultados observados mostram que os diferentes tipos de dieta não influenciaram esses parâmetros, enquanto que o estresse térmico proporcionou menor $(\mathrm{P}<0,05)$ valor de $\mathrm{pH}$ final (Tabela 5). Isto pode estar relacionado ao aumento da velocidade do desenvolvimento do rigor mortis, causado pelo estresse térmico préabate e esgotamento das reservas de glicogênio pela sua conversão a lactato (McKEE; SAMS, 1997). Entretanto os valores de $\mathrm{pH}$ inicial, intensidade de luminosidade, intensidade de vermelho e amarelo não foram influenciados pelo tipo de dieta e pelo estresse térmico.

Tabela 5. Valores de $\mathrm{pH}$ inicial e final, luminosidade (L), intensidade de vermelho ( $\left.\mathrm{a}^{*}\right)$ e intensidade de amarelo $\left(\mathrm{b}^{*}\right)$ de músculos Pectolares major de frangos de corte submetidos a diferentes dietas no período terminação e submetidos a estresse térmico de $35^{\circ} \mathrm{C}$ por 30 minutos.

\begin{tabular}{llllll}
\hline Tratamento & $\mathrm{pH}$ inicial & $\mathrm{pH}$ final & $\mathrm{L}$ & $\mathrm{a}^{*}$ & $\mathrm{~b}^{*}$ \\
\hline Tipo de ração (R) & & & & & \\
Controle & 6,14 & 5,95 & 53,74 & 0,79 & 8,60 \\
Sem supl.vit-mineral & 6,19 & 6,00 & 53,93 & 0,62 & 8,00 \\
Sem fosfato e calcário & 6,17 & 5,99 & 53,05 & 0,94 & 8,93 \\
\hline Teste F & $0,29^{\mathrm{NS}}$ & $0,45^{\mathrm{NS}}$ & $0,61^{\mathrm{NS}}$ & $1,41^{\mathrm{NS}}$ & $2,40^{\mathrm{NS}}$ \\
DMS & 0,15 & 0,12 & 1,99 & 0,45 & 1,03 \\
\hline Manejo pré-abate (E) & & & & & \\
Sem estresse & 6,21 & $6,03 \mathrm{a}$ & 53,49 & 0,84 & 8,73 \\
Com estresse & 6,12 & $5,94 \mathrm{~b}$ & 53,66 & 0,73 & 8,29 \\
\hline Teste F & $3,87^{\mathrm{NS}}$ & $4,71^{*}$ & $0,62^{\mathrm{NS}}$ & $0,50^{\mathrm{NS}}$ & $1,63^{\mathrm{NS}}$ \\
DMS & 0,10 & 0,8 & 1,35 & 0,31 & 0,70 \\
\hline F interação R x E & $0,52^{\mathrm{NS}}$ & $1,47^{\mathrm{NS}}$ & $0,58^{\mathrm{NS}}$ & $0,97^{\mathrm{NS}}$ & $0,80^{\mathrm{NS}}$ \\
Coef. Variação (\%) & 3,47 & 2,90 & 5,36 & 83,55 & 17,45 \\
\hline
\end{tabular}

Na mesma coluna, médias seguidas de letras diferentes, indicam diferenças estatísticas pelo teste de Tukey $(\mathrm{P}<0,05)$; DMS $=$ diferença mínima significativa.

Fonte: Elaboração dos autores. 
Os resultados das análises de capacidade de retenção de água, perdas por cozimento e força de cisalhamento não foram influenciados pelos diferentes tratamentos nutricionais (Tabela 6), entretanto a promoção de estresse pré-abate proporcionou maiores perdas na cocção da carne em relação ao manejo sem estresse. Isto ocorreu em função de que o estresse pré-abate proporciona aumento da temperatura corporal e glicólise mais rápida, proporcionando após a morte do animal queda do $\mathrm{pH}$ mais pronunciada, a uma temperatura elevada, o que causa desnaturação proteica (OLIVO et al., 2001). Isso resulta em uma menor capacidade de retenção de água, maior perda por cozimento e uma textura mais dura da carne. Porém, pelos resultados obtidos, apenas foi observado diferenças significativas para a perda por cocção, que foram maiores nas aves estressadas termicamente. Com relação a interação entre os tipos de rações e os manejos pré-abate, não foram observadas interações para as análises que avaliam a qualidade de carne.

Tabela 6. Valores de capacidade retenção água (CRA), perdas de cozimento (PC) e força de cisalhamento (FC) de peitos de frangos alimentados com diferentes dietas e submetidos ou não ao estresse térmico pré-abate.

\begin{tabular}{llcl}
\hline Tratamento & CRA $(\%)$ & PC $(\%)$ & FC $\left(\mathrm{N} / \mathrm{cm}^{2}\right)$ \\
\hline Tipo de ração (R) & & & \\
Controle & 70,75 & 26,30 & 18,34 \\
Sem Premix & 68,90 & 26,00 & 17,41 \\
Sem Ca e P & 69,49 & 26,98 & 20,61 \\
\hline Teste F & $1,29^{\mathrm{NS}}$ & $0,70^{\mathrm{NS}}$ & $1,73^{\mathrm{NS}}$ \\
DMS & 2,89 & 2,07 & 4,36 \\
\hline Manejo pré-abate (E) & & & \\
Sem estresse & 70,11 & $25,72 \mathrm{~b}$ & 17,62 \\
Com estresse & 69,32 & $27,13 \mathrm{a}$ & 19,95 \\
\hline Teste F & $0,67^{\mathrm{NS}}$ & $4,25^{*}$ & $2,63^{\mathrm{NS}}$ \\
DMS & 1,96 & 1,40 & 4,36 \\
\hline F interação R x E & $0,41^{\mathrm{NS}}$ & $0,91^{\mathrm{NS}}$ & $0,38^{\mathrm{NS}}$ \\
\hline Coef. Variação (\%) & 4,12 & 7,76 & 23,02 \\
\hline
\end{tabular}

$\mathrm{Na}$ mesma coluna, médias seguidas de letras diferentes, indicam diferenças estatísticas pelo teste de Tukey $(\mathrm{P}<0,05)$; DMS $=$ diferença mínima significativa.

Fonte: Elaboração dos autores.

\section{Conclusões}

Pelos resultados do presente trabalho pode-se concluir que a retirada do suplemento vitamínico mineral ou o fosfato bicálcico e o calcário da dieta de frangos na fase de terminação não prejudicou o desempenho, características da carcaça e cortes e os níveis sanguíneos de Ca e P, mostrando-se assim uma possível forma de reduzir os custos de produção. O estresse térmico quente no pré-abate proporcionou uma redução na qualidade de carne.

\section{Referências}

BARBUT, S. Estimates and detection of the PSE problem in young trukey breast meat. Canadian Journal of Animal Science. Ottawa, v. 76, n. 3, p. 455-457, 1996.

CASON, J. A.; LYON, C. E.; PAPA, C. M. Effect of muscle oppositon during rigor on development of broiler breast meat tenderness. Poultry Science, Champaigh, v. 76, n. 5, p. 725-787, 1997.

CHRISTMAS, R. B.; HARMS, R. H.; SLOAN, D. R. The absence of vitamins and trace minerals and broiler performance. Journal Applied Poultry Research, Champaigh, v. 4, n. 4, p. 407-410, 1995. 
HALLBERG, L.; ROSSANDER-HULTÉN, L.; BRUNE, M.; GLEERUP, A. Calcium and iron absorption: mechanism of action and nutritional importance. European Journal of Clinical Nutrition, London, v. 46, n. 5, p. 317-27, 1992.

KHAJALI, F.; ASADI, K. E.; ZAMANI, M. Effect of vitamin and trce mineral withdrawal from finisher diets on growth performance and immunocompetence of broiler chickens. British Poultry Science, London, v. 47, n. 2, p. 159-162, 2006.

MAIORKA, A.; LAURENTIZ, A. C.; SANTIN, E.; ARAUJO, L. F.; MACARI, M. Dietary vitamino or mineral mix removal during the finisher period on broiler chicken performance. Journal Applied Poultry Research, Champaigh, v. 11, n. 2, p. 121-126, 2002.

MCKEE, S. R.; SAMS, A. R. The effect of seasonal heat stress on rigor development and the incidence of pale, exudative turkey meat. Poultry Science, Champaigh, v. 76, n. 11, p. 1616-1620, 1997.

MORAVEJ, H.; ALAHYARI-SHAHRASB, M.; BAGHANI, M. R.; SHIVAZAD, M. Withdrawal or reduction of the dietary vitamin premix on bone parameters of broiler chickens in two rearing systems. South African Journal of Animal Science, Pretoria, v. 42, n. 2, p. 169-177, 2012.

OLIVO, R.; SOARES, A. L.; IDA, E. I.; SHIMOKOMAKI, M. Dietary vitamin E inhibits poultry PSE and improves meat functional properties. Journal of Food Biochemistry, Oxford, v. 25, n. 4, p. 271-283, 2001.

PISSINATI, A.; MEDEIROS, L. G.; OBA, A.; ALMEIDA, M.; SPIRANDELLI, T. C.; CARVALHO, L. D. P. Influence of organic selenium supplementation over yield grade, performance and meat quality of broilers. In: WORLD'S POULTRY SCIENCE, 2012, Salvador. Anais...Salvador, 2012. 1 CD-ROM.
ROSTAGNO, H. S.; ALBINO, L. F. T.; DONZELE, J. L.; GOMES, P. C.; OLIVEIRA, R. F.; LOPES, D. C.; FERREIRA, A. S.; BARRETO, S. L. T. Tabelas brasileiras para aves e suínos: composição de alimentos e exigências nutricionais. 2. ed. Viçosa: UFV, 2005. 186 p.

SÁ, L. M.; GOMES, P. C.; ROSTAGNO, H. S.; ALBINO, L. F. T.; CECON, P. R.; D`AGOSTINI, P. Exigência nutricional de cálcio para frangos de corte, nas fases de crescimento e terminação. Revista Brasileira de Zootecnia, Viçosa, v. 33, n. 2, p. 397-406, 2004.

SAYADI, A. J.; NAVIDSHAD, B.; ABOLGHASEMI, A.; ROYAN, M.; SEIGHALANI, R. Effects of dietary mineral premix reduction or withdrawal on broilers performance. International Journal of Poultry Science, Pakistan, v. 4, n. 11, p. 896-899, 2005.

SCHEIDELER, S. E.; RIVES, D. V.; GARLICH, J. D.; FERKET, P. R. Dietary calcium and phosphorus effects on broiler performance and the incidence of sudden death syndrome mortality. Poultry Science, Champaigh, v. 74, n. 12, p. 2011-2018, 1995.

SKINNER, J. E.; WALDROUP, A.; WALDROUP, P. W. Effects of removal of vitamin and trace mineral supplements from grower and finisher diets on live performance and carcass composition of broilers. Journal Applied Poultry Research, Champaigh, v. 1, n. 3, p. 280286, 1992. 